\title{
Transferts du radiocobalt en milieu terrestre*
}

\author{
C. COLLE**, S. ROUSSEL DEBET**, J. REAL**
}

(Manuscrit reçu le 18 juillet 1995, révisé le 19 décembre 1995, accepté le 7 mai 1996)

RÉSUMÉ Un bilan résumé des connaissances sur le radiocobalt dans le milieu terrestre est présenté. La chimie du cobalt dans les sols est étroitement liée à celle du fer et du manganèse. Il présente une meilleure solubilité en milieu acide. On estime que 5 à $10 \%$ du cobalt total du sol sont hydrosolubles ou facilement extractibles, et que la majeure partie du cobalt est, soit faiblement adsorbée sur les argiles, soit complexée avec les acides humiques. L'intensité de l'absorption racinaire du cobalt par les végétaux est conditionnée principalement par le pH du sol, par la présence d'éléments tels le fer, l'aluminium ou le calcium et, dans une moindre mesure, par la texture du sol et sa teneur en matière organique. Les résultats concernant le transfert foliaire sont peu nombreux. Pour une contamination foliaire unique, l'intensité du transfert varie en fonction du stade végétatif de la plante au moment de la contamination. Comme pour d'autres radioéléments, le transfert résultant d'un apport sur les organes aériens est plus pénalisant que le transfert d'origine racinaire, pour un dépôt équivalent. Les transformations alimentaires permettent d'abaisser de près de $50 \%$ l'activité résiduelle des aliments, par comparaison avec les végétaux récoltés bruts.

ABSTRACT This paper presents a summarized review of the knowledge relative to the behaviour of radiocobalt in the terrestrial environment. The chemistry of cobalt in soils is closely connected to that of iron and manganese. It is more soluble in acidic conditions. In soils, 5 to $10 \%$ of total cobalt may be hydrosoluble or easily extractible, and the main part of cobalt may be either weakly adsorbed on clays or complexed with humic acids. The importance of cobalt absorption by the roots of plants depends mainly on the pH of the soil, on the presence of other elements such as iron, aluminium or calcium and to a lesser extent on the soil texture and organic matter content. Results concerning foliar transfer are sparse and very variable. For a single foliar contamination it depends on the vegetative stage of the plant at the time of deposit. As for other elements, the transfer resulting from a deposit onto aerial parts of the plants is more important than the root uptake, for a similar deposition. In comparison to raw products, food processing results in lowering the residual activity of foods down to $50 \%$.

\footnotetext{
* Ce texte fait partie d'une série d'articles de synthèse consacrés à la dosimétrie et qui sera publiée durant l'année 1996.

** Institut de Protection et de Sûreté Nucléaire, Département de Protection de l'Environnement et des Installations, Service d'Etudes et de Recherches sur les transferts dans l'Environnement, Laboratoire de radioécologie continentale, Centre de Cadarache, $13108 \mathrm{St}$ Paul Lez Durance, France.
} 


\section{Introduction}

Les radiocobalts sont des produits d'activation présents dans les effluents liquides des réacteurs électronucléaires et des usines de retraitement des combustibles. Ils ont, par conséquent, fait l'objet d'études radioécologiques destinées à évaluer les risques potentiels que pouvait engendrer, pour l'homme, leur présence dans l'environnement. Toutefois, ils n'ont pas marqué de façon significative les écosystèmes terrestres du fait de leur absence dans les rejets atmosphériques de routine des installations nucléaires ainsi que dans les retombées des tirs d'essais d'armes. En outre, le marquage en radiocobalt résultant de l'accident de Tchernobyl a été insignifiant au delà de l'environnement proche du réacteur accidenté.

De ce fait, les connaissances relatives au transfert du radiocobalt en milieu terrestre résultent uniquement d'études expérimentales. La validité des résultats obtenus sur les modèles réduits de laboratoire a été corroborée par la réalisation d'essais sur lysimètres de grande taille.

\section{Caractéristiques générales du cobalt}

Le cobalt, de numéro atomique $Z=27$, est un élément de transition qui possède une masse atomique de 58,9. Dix isotopes radioactifs (54 à 64) et cinq isomères nucléaires sont connus. Le plus important d'entre eux, en radioécologie terrestre, est le cobalt 60 (émetteur gamma, période : 5,26 ans) qui appartient au groupe 2 (radiotoxicité forte).

D'un point de vue chimique le cobalt possède deux valences principales (II et III), le degré II étant le plus stable, mais il peut être monovalent dans certains composés organiques (Pascal, 1961).

La concentration moyenne en cobalt de l'écorce terrestre est estimée à $23 \mathrm{mg} \mathrm{kg}^{-1}$ (trente-troisième élément par ordre d'abondance). Les concentrations les plus fortes sont mesurées dans les roches très riches en magnésium et les plus faibles dans les roches calcaires et les grès (Tab. I).

Les teneurs en cobalt des sols sont généralement du même ordre de grandeur que celles des roches mères dont ils sont issus et varient en général de 3 à $15 \mathrm{mg} \mathrm{kg}^{-1}$ (Coughtrey, 1983).

\section{Comportement du cobalt dans les sols}

De nombreux travaux ont eu pour objet l'étude du comportement du cobalt dans les sols, notamment à partir d'expériences d'extraction du cobalt du sol (Alban et Kubota, 1960 ; Kerpen, 1986 ; Lopez et Graham, 1972). 
TABLEAU I

Abondance naturelle du cobalt dans quelques roches mères, selon Coughtrey (1983) et Loué (1986)

Natural concentration of cobalt in some parent materials, from Coughtrey (1983) and Loué (1986)

\begin{tabular}{|llc|}
\hline \multicolumn{2}{|c|}{ Roches meres } & Concentration en mg kg-1 \\
\hline \multirow{2}{*}{ sédimentaires } & Calcaires & 1 à 5 \\
& Grès & 1 à 50 \\
& Schistes & 15 à 20 \\
ignées & Granites & 5 à 10 \\
& Basaltés & 35 à 45 \\
& Ultrabasiques riches en $\mathrm{Mg}$ & 100 à 300 \\
\hline
\end{tabular}

Le comportement du cobalt dans les sols s'apparente - et apparait très lié à celui du manganèse et du fer ; il existe une corrélation entre la concentration en cobalt total du sol et celle en manganèse total, $80 \%$ du cobalt étant associé avec des oxydes de manganèse (Taylor, 1968). Le cobalt présente une meilleure solubilité en milieu acide et, dans ce cas, la forme chimique dominante est l'ion divalent $\mathrm{Co}^{2+}$. En condition neutre ou légèrement alcaline c'est le cation $\mathrm{Co}(\mathrm{OH})_{2}{ }^{+}$qui prédomine. A partir du $\mathrm{pH} 8$, les hydroxydes de cobalt commencent à précipiter. Il en résulte que la mobilité du cobalt dans les sols diminue lorsque le $\mathrm{pH}$ augmente. Le cobalt présente également une certaine affinité pour les oxydes de fer ce qui explique sa plus grande mobilité en milieu réducteur, notamment dans les sols hydromorphes (Coughtrey, 1983).

A l'état $\mathrm{Co}^{2+}$ (rayon ionique : 0,72 $\AA$, coordinence théorique : 6) le cobalt peut faire partie des cations constitutifs des argiles dans une proportion d'environ $3 \%$ de la capacité d'échange cationique. Toutefois, il se trouverait en majeure partie (80 à $90 \%$ ) soit faiblement adsorbé sur les argiles en liaison avec les oxydes de fer et de manganèse, soit complexé par les acides humiques. De ce fait, on considère que seulement 5 à. $10 \%$ du cobalt total d'un sol seraient biodisponibles (Taylor, 1968 ; Tiller et al., 1962).

La migration en profondeur de l'élément dépend évidemment des caractéristiques du sol, et dans les sols à faible capacité d'échange, un lessivage modéré est à prévoir. En revanche, les sols ou les horizons riches en matière organique ont tendance à fixer fortement et durablement le cobalt. On estime à environ $5 \%$ par an la fraction de cobalt qui peut être remobilisée pour la migration vers les horizons minéraux du sol (Schimmack et al., 1994), et la période (demi-vie) moyenne de résidence dans la litière varie de 2 à 12 mois (Coughtrey, 1983) selon la végétation.

L'ordre de grandeur des coefficients de partage $\mathrm{Kd}$ du cobalt dans les sols est présenté (Tab. II) à partir d'une compilation bibliographique (Thibault, 
1990). Dans cette base de données sont rassemblées les résultats relatifs aux sols, aux composants minéralogiques, aux milieux géologiques profonds, ce qui explique les intervalles de variation importants. Pour les valeurs répertoriées sous l'étiquette "sol limoneux" qui correspondent à des sols de type agricole, le Kd moyen est de $1300 \mathrm{dm}^{3} \mathrm{~kg}^{-1}$, ce qui dénote une fixation assez forte, ce que confirment des expérimentations (Baes et Sharp, 1981 ; Bunzl et Schimmack, 1988) effectués par contamination in situ de sols bruns.

TABLEAU II

Valeurs ( $\mathrm{dm}^{3} \mathrm{~kg}^{-1}$ ) du coefficient Kd de partage eau-sol pour le cobalt, selon Thibault (1990)

Values ( $\mathrm{dm}^{3} \mathrm{~kg}^{-1}$ ) of soil-water distribution coefficient $\mathrm{Kd}$ of cobalt, from Thibault (1990)

\begin{tabular}{|c|c|c|c|c|}
\hline \multicolumn{5}{|c|}{ 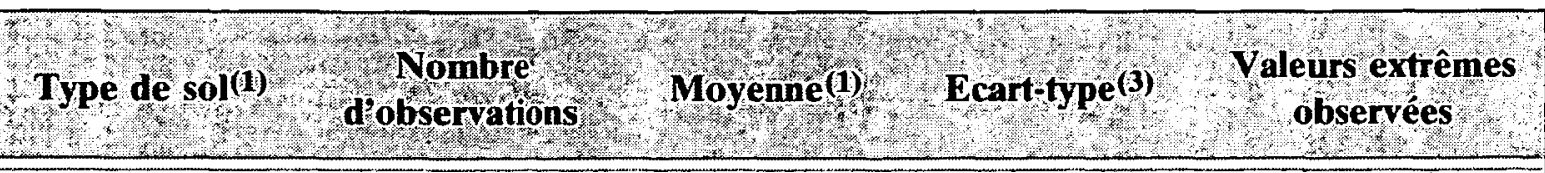 } \\
\hline sableux & 33 & 60 & 2,8 & 0,07 à 9000 \\
\hline limoneux & 23 & 1300 & 1,3 & 100 à 9700 \\
\hline argileux & 15 & 550 & 1,8 & 20 à 14000 \\
\hline organique & 6 & 1000 & 1,5 & 120 à 4500 \\
\hline
\end{tabular}

(1) Classement de la référence "sableux" : $\geq 70 \%$ de sables ; "limoneux" : texture équilibrée (1/3 de sables, $1 / 3$ de limons, $1 / 3$ d'argiles) ou $\geq 80 \%$ de limons; "argileux" : $\geq 35 \%$ d'argiles ; "organique" : $30 \%$ de matière organique.

(2) Moyenne géométrique des observations, la distribution étant supposée Log-Normale.

(3) Ecart-type géométrique des observations.

\section{Le cobalt dans les végétaux}

\subsection{Rôle physiologique et teneur dans les végétaux}

Le cobalt est un oligo-élément, indispensable à très faible concentration (10 $\mu \mathrm{g} \mathrm{l}^{-1}$ dans la solution du sol) à la fixation symbiotique de l'azote par le Rhyzobium dans les nodules racinaires des légumineuses. Il intervient dans la formation du coenzyme B12 nécessaire aux bactéries, mais les mécanismes de son action ne sont pas encore clairement définis. Son importance pour les autres végétaux n'est pas élucidée ; il semblerait qu'il intervienne dans les mécanismes enzymatiques du métabolisme des glucides (Loué, 1986).

Les teneurs moyennes en cobalt rencontrées dans les plantes varient de 0,1 à $1 \mathrm{mg} \mathrm{kg}^{-1}$ dans la matière sèche, les concentrations des légumineuses étant plus fortes que celles des graminées. Sur certains sols issus de la dégradation de roches métamorphiques, serpentine en particulier, les concentrations de cobalt mesurées' dans les végétaux peuvent atteindre $100 \mathrm{mg} \mathrm{kg}^{-1}$. Des teneurs importantes $\left(800\right.$ à $\left.1000 \mathrm{mg} \mathrm{kg}^{-1}\right)$ ont été observées chez certaines espèces : 
Crotolaria cobalticola (Zaïre), Nyssa sylvatica (Sud-Est des Etats Unis) (Loué, 1986). D'autre part, à proximité d'exploitations minières, ou sur des zones de décharges industrielles, des symptômes de toxicité ont été observés, les dicotylédones s'avérant plus sensibles que les monocotylédones (Coughtrey, 1983).

\subsection{Les relations sol - plante et les facteurs de transfert racinaires}

Conventionnellement, on quantifie l'intensité d'absorption racinaire d'un radioélément par un végétal à l'aide du "facteur de transfert sol-plante" (ou "facteur de transfert racinaire") qui est le rapport de l'activité massique de la plante à l'activité massique du sol, la répartition du radioélément étant supposée homogène dans la couche de sol de 0 à $20 \mathrm{~cm}$. L'activité massique du végétal peut être exprimée par rapport au poids sec ou frais, alors que l'activité du sol est toujours relative au poids sec.

L'absorption racinaire du cobalt par les végétaux est conditionnée essentiellement par les caractéristiques physico-chimiques du sol (D'Souza et Mistry, 1979 ; Lambrechts et al., 1988). Le paramètre le plus important est le pH qui, par son action sur la solubilité de cet élément dans le sol, affecte de façon très nette son absorption qui augmente avec l'acidité du milieu. La présence d'éléments tels le fer, l'aluminium ou le calcium joue également un rôle sur l'absorption du cobalt par les végétaux, ceci résultant plus probablement de la compétition entre ces ions au niveau du sol que d'interactions au niveau des mécanismes physiologiques d'assimilation des plantes. Dans une moindre mesure, la texture du sol et sa teneur en matière organique font également partie des paramètres qui peuvent influencer les transferts sol-plante du cobalt.

Une expérimentation (Colle et al., 1991) montre que l'apport de $50 \mathrm{mg} \mathrm{kg}-1$ de cobalt stable au sol entraîne une augmentation du facteur de transfert racinaire d'un facteur 2 à 4 , probablement par effet d'entraînement.

De nombreuses études expérimentales ont été effectuées pour évaluer les facteurs de transfert sol-plante du cobalt 60 . Les données qui en résultent ont été rassemblées par l'Union Internationale des Radioécologistes, qui en a déduit, par analyse statistique, des valeurs recommandées exprimées en $\mathrm{kg}$ de sol sec $\mathrm{kg}^{-1}$ de végétal sec (Tab. III). Celles-ci sont comprises entre 0,2 pour les légumes verts et 0,004 pour les grains de céréales. L'incertitude pour un même type de végétal est de l'ordre d'un facteur 2 ; elle peut néanmoins s'avérer beaucoup plus élevée en raison de la grande diversité des conditions expérimentales et des types de sols utilisés.

\subsection{Transfert par voie foliaire}

Les quelques résultats concernant le transfert foliaire résultant d'un apport chronique par aspersions répétées de solutions de radiocobalt sur les parties aériennes de végétaux montrent que le pourcentage retenu par les parties 
aériennes des végétaux à la récolte est moins de $1 \%$ de l'apport total pour des légumes (haricots verts, salades). (Real et al., 1983), alors qu'il atteint $50 \%$ pour des céréales (Coughtrey, 1983).

Lorsque la contamination foliaire résulte d'un apport unique, les résultats sont très variables selon le stade végétatif lors de la contamination. Des expérimentations sur les céréales contaminées par aspersion à divers stades végétatifs (Coughtrey, 1983) indiquent que le pourcentage initial d'activité retenu par les organes aériens du végétal augmente avec la biomasse au moment de la contamination, en raison de l'interception par les parties aériennes lors du dépôt. En outre, le pourcentage d'activité présent dans le grain à la récolte, qui résulte de la translocation à partir de l'activité interceptée, est lié au délai séparant la récolte de la contamination (Fig. 1) : lorsque la contamination a lieu pendant la période de formation du grain, l'activité du grain récolté est maximale, alors qu'elle est plus faible si la contamination a lieu à des stades végétatifs plus proches de la récolte, celle de la paille, au contraire, augmentant fortement. La translocation de l'activité interceptée vers le grain s'effectuerait donc essentiellement durant la courte période d'élaboration des grains.

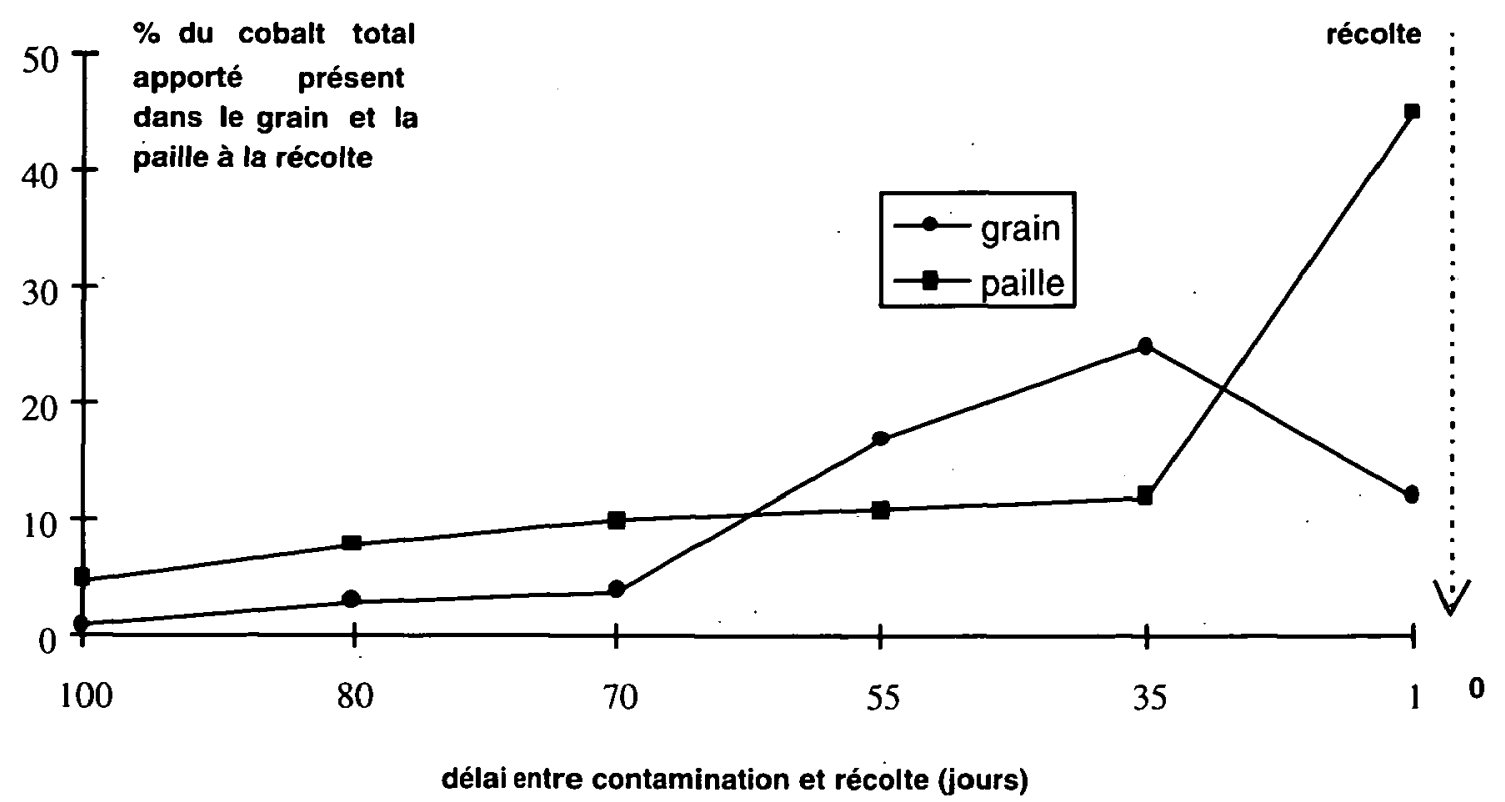

Fig. 1 - Influence du délai entre la contamination et la récolte d'une céréale (orge) sur l'activité du grain et de la paille lors de la récolte, selon Coughtrey (1983)

Effect of the delay between contamination and harvest of the cereal (barley) on the activity of grain and straw at the time of harvest, from Coughtrey (1983) 


\section{TABLEAU III}

Valeurs des facteurs de transfert sol-plante du cobalt ( $k g$ sol sec $\mathrm{kg}^{-1}$ végétal sec), selon l'UIR (1989) Values of soil-to-plant transfer factors of cobalt (kg dry soil $\mathrm{kg}^{-1}$ dry plant) from l'UIR (1989)

\begin{tabular}{|c|c|c|c|c|}
\hline Wregetal & $\begin{array}{l}\text { Nomblo } \\
\text { Sserve } \\
\end{array}$ & $\begin{array}{l}\text { Valeus } \\
\text { ommand } \\
p\end{array}$ & eritu & $\begin{array}{l}\text { Intervalle } \\
\text { de variation(3) }\end{array}$ \\
\hline Orge grain & 32 & $2,4 \mathrm{E}-3$ & 2,0 & $1,2 \mathrm{E}-3-4,8 \mathrm{E}-3$ \\
\hline Maïs grain & 2 & $1,9 \mathrm{E}-2$ & - & - \\
\hline Blé grain & 28 & $5,7 \mathrm{E}-3$ & 2,0 & $2,8 \mathrm{E}-3-1,2 \mathrm{E}-2$ \\
\hline Céréales & 62 & 3,7E-3 & 2,0 & $1,9 \mathrm{E}-3-7,4 \mathrm{E}-3$ \\
\hline Luzerne fourrage & 4 & $1,1 \mathrm{E} 0$ & 3,8 & $2,9 \mathrm{E}-1-4,2 \mathrm{E} 0$ \\
\hline Trèfle fourrage & 32 & $9,4 \mathrm{E}-2$ & 2,0 & $4,7 E-2-1,9 \mathrm{E}-1$ \\
\hline Maïs fourrage & 96 & $1,9 \mathrm{E}-2$ & 2,0 & $9,5 \mathrm{E}-3-3,8 \mathrm{E}-2$ \\
\hline Herbe & 112 & $5,4 \mathrm{E}-2$ & 2,0 & $2,7 \mathrm{E}-2-1,1 \mathrm{E}-1$ \\
\hline Fourrages & 132 & $3,1 \mathrm{E}-2$ & 2,0 & $1,6 \mathrm{E}-2$ - $6,2 \mathrm{E}-2$ \\
\hline Haricot gousse & 138 & $3,0 \mathrm{E}-2$ & 2,0 & $1,5 \mathrm{E}-2-6,0 \mathrm{E}-2$ \\
\hline Carotte racine & 2 & $1,3 \mathrm{E}-1$ & - & - \\
\hline Radis racine & 8 & $1,2 \mathrm{E}-1$ & 2,4 & $5,0 \mathrm{E}-2-2,9 \mathrm{E}-1$ \\
\hline Pomme de terre & & & & \\
\hline tubercule & 64 & $6,0 \mathrm{E}-2$ & 2,0 & $3,1 \mathrm{E}-2-1,2 \mathrm{E}-1$ \\
\hline Choux & 33 & $4,4 \mathrm{E}-2$ & 2,0 & $2,2 \mathrm{E}-2-8,8 \mathrm{E}-2$ \\
\hline Laitue & 4 & $2,8 \mathrm{E}-1$ & 3,8 & $7,4 \mathrm{E}-2-1,1 \mathrm{E} 0$ \\
\hline Epinards & 129 & 2,9E-1 & 2,0 & $1,5 \mathrm{E}-1-5,8 \mathrm{E}-1$ \\
\hline Légumes verts (4) & 166 & 2,0E-1 & 2,0 & $1,0 \mathrm{E}-1$ - 4,0E-1 \\
\hline
\end{tabular}

(1) $\mu$ : moyenne géométrique des observations, la distribution étant supposée Log-Normale.

(2) $I$ : facteur d'incertitude sur la moyenne des observations, au niveau de confiance de $95 \%$.

La valeur de $I$ est calculée par : $\exp \left(2 \sigma /(N-1)^{0,5}\right)$, avec $\sigma$ : écart-type des Logarithmes des $N$ observations, si $I>2$, sinon, la valeur de $I$ est prise égale à 2 , pour des raisons explicitées par l'UIR.

(3) Intervalle calculé par $\mu / I$ et $\mu \times I$

(4) Légumes verts : choux, laitue, épinards.

\subsection{Importance relative des voies de transfert aux végétaux}

Peu d'études permettent la comparaison directe de l'importance relative des transferts racinaire et foliaire du radiocobalt. Une expérimentation effectuée au S.E.R.E(1) de 1985 à 1990 sur 16 bacs contenant $200 \mathrm{~kg}$ de sol brun calcaire (Colle et al., 1983, 1991) met en évidence l'importance des modalités de contamination sur le transfert du cobalt 60 aux végétaux (Tab. IV).

(1) Service d'études et de recherche sur les transferts dans l'environnement. 
Pour un même dépôt surfacique sur le sol ou la végétation, le transfert par voie foliaire est de loin le plus pénalisant pour la contamination de la chaîne alimentaire, comme c'est le cas pour le césium et le strontium. Le transfert par voie racinaire est inférieur de deux à trois ordres de grandeur à l'absorption par voie foliaire.

Le dépôt en surface du sol entraîne une augmentation du transfert par rapport à un apport incorporé de façon homogène d'environ un facteur 3 , pour la première culture.

Après une première récolte sur un sol contaminé par un dépôt de surface, un labour superficiel augmente légèrement le transfert à la culture suivante, alors qu'un labour normal, voire profond est assez efficace pour diminuer le transfert. Cet effet, dont l'efficacité dépend de la profondeur du système racinaire, s'atténue dans le temps pour les récoltes successives de luzerne. Enfin, dans les conditions de l'expérimentation, le fractionnement des apports n'a pas d'effet significatif.

\section{TABLEAU IV}

Influence du mode d'apport et des labours sur le facteur de transfert global du cobalt $\left(\mathrm{m}^{2} \mathrm{~kg}^{-1}\right.$ de végétal sec) selon Colle et al. (1991)

Effect of the contamination mode and of tillage on the global transfer factor of cobalt ( $\mathrm{m}^{2} \mathrm{~kg}^{-1}$ dry plant), from Colle et al. (1991)

\begin{tabular}{|c|c|c|c|}
\hline Mode de contamination & (graé & $\begin{array}{l}\text { Carot } \\
\text { (racin } \\
\end{array}$ & 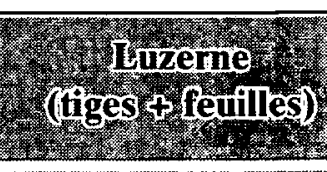 \\
\hline Dépôt sur sol nu & & & \\
\hline Contamination homogène(1) en profondeur & $2,7 \bar{E}-5$ & $1,2 \mathrm{E}-4$ & $6,0 \mathrm{E}-5$ \\
\hline Dépôt en surface du sol(2) & & & \\
\hline $\begin{array}{ll}\text { 1re }^{\text {culture }} & 1 \text { apport } \\
& 3 \text { apports }\end{array}$ & $\begin{array}{l}6,0 \mathrm{E}-5 \\
4,7 \mathrm{E}-5\end{array}$ & $3,3 \mathrm{E}-3$ & $2,0 \mathrm{E}-4$ \\
\hline $\begin{aligned} 2^{e} \text { culture après labour } & \text { superficiel }(10 \mathrm{~cm}) \\
& \text { normal }(30 \mathrm{~cm}) \\
& \text { profond }(50 \mathrm{~cm})\end{aligned}$ & $\begin{array}{l}8,7 \mathrm{E}-5 \\
1,7 \mathrm{E}-5 \\
3,3 \mathrm{E}-6\end{array}$ & & $\begin{array}{c}2,7 \mathrm{E}-4 \\
1,3 \mathrm{E}-5 \text { à } 7,3 \mathrm{E}-5(4) \\
3,3 \mathrm{E}-6 \text { à } 4,0 \mathrm{E}-5(4)\end{array}$ \\
\hline $\begin{array}{r}\text { Dépôt sur couvert végétal(3) } \\
\text { unique } \\
3 \text { apports }\end{array}$ & $\begin{array}{l}1,1 \mathrm{E}-2 \\
1,1 \mathrm{E}-2\end{array}$ & $1,8 \mathrm{E}-1$ & \\
\hline
\end{tabular}

(1) Contamination homogène des 30 premiers centimètres du sol.

(2) Pulvérisation de solution contaminée sur le sol nu.

(3) Pulvérisation de solution contaminée sur la partie aérienne des végétaux.

(4) Respectivement $1^{\text {re }}$ et $7^{\mathrm{e}}$ récolte. 


\section{Effet des transformations alimentaires}

Comme pour d'autres radioéléments, les concentrations en cobalt des aliments dépendent des processus de transformation appliqués aux récoltes. Le tableau $\mathrm{V}$ indique pour quelques transformations les résultats obtenus sur les principales productions. Les préparations culinaires courantes de végétaux (lavage, cuisson) et les transformations alimentaires permettent d'abaisser notablement l'activité des aliments transformés. Par exemple, dans l'hypothèse d'une contamination d'un sol de $100 \mathrm{~Bq} \mathrm{~kg}^{-1} \mathrm{sec}$, l'activité massique susceptible d'être retrouvée dans un légume-fruit tel que le haricot vert ayant poussé sur ce sol serait de l'ordre de $0,3 \mathrm{~Bq} \mathrm{~kg}{ }^{-1}$ frais (compte-tenu d'une teneur en matière sèche du végétal de $10 \%$ ). Après mise en conserve, l'activité massique du produit consommable serait de $0,12 \mathrm{~Bq} \mathrm{~kg}^{-1}$ frais (hors décroissance radioactive) ; la même estimation pour une salade (consommée crue) aboutirait à une activité massique finale de $1,2 \mathrm{~Bq} \mathrm{~kg}^{-1}$ frais d'aliment.

TABLEAU V

Effet des transformations alimentaires sur le transfert du radiocobalt selon Colle (1994)

Effect of food processing on the radiocobalt transfer, from Colle (1994)

\begin{tabular}{|llll|}
\hline Produift & \multicolumn{1}{c}{ Transformation } & $\boldsymbol{R}^{(\mathbf{1})}$ & $\boldsymbol{F t}^{(2)}$ \\
\hline \multirow{2}{*}{ Céréales } & Blé dur $\rightarrow$ semoule & 0,75 & 0,60 \\
& Blé tendre $\rightarrow$ farine & 0,75 & 0,20 \\
& Riz rond $\rightarrow$ riz blanchi & 0,88 & 0,60 \\
& Riz long $\rightarrow$ riz blanchi & 0,88 & 0,40 \\
\hline Légumes & Petit pois $\rightarrow$ conserve & 1,10 & 0,20 \\
& Haricot vert $\rightarrow$ conserve & 1,00 & 0,40 \\
& Tomate $\rightarrow$ conserve & 0,97 & 0,30 \\
& Carotte $\rightarrow$ conserve & 0,60 & 0,10 \\
\hline Fruits & Cerise $\rightarrow$ conserve au sirop & 0,90 & 0,50 \\
& Pêche $\rightarrow$ conserve au sirop & 0,88 & 0,40 \\
& Poire $\rightarrow$ conserve au sirop & 0,88 & 0,80 \\
\hline Vin & Raisin $\rightarrow$ vin rosé & 0,45 & 0,50 \\
& Raisin (grappes) $\rightarrow$ vin rouge & 0,50 & 0,60 \\
& Raisin (grains) $\rightarrow$ vin rouge & 0,43 & 0,30 \\
& Vin $\rightarrow$ alcool & 0,10 & 0,00 \\
\hline
\end{tabular}

(1) $R: \mathrm{kg}$ frais de produit transformé / $\mathrm{kg}$ frais de produit brut.

(2) $F t: \mathrm{Bq} \mathrm{kg}^{-1}$ frais de produit transformé / $\mathrm{Bq} \mathrm{kg}^{-1}$ frais de produit brut. 


\section{Conclusion}

La quasi absence de radiocobalt dans les rejets atmosphériques dés installations nucléaires françaises et dans les retombées issues des explosions aériennes ou des accidents ayant affecté le territoire national se traduit en France par l'absence de marquage du milieu terrestre. Seule l'irrigation ou l'inondation de parcelles pourrait conduire à la présence de radiocobalt dans les sols et les cultures, vu la présence des isotopes 57, 58 et 60 dans les effluents liquides des réacteurs à eau pressurisée et des usines de retraitement françaises.

Les données acquises proviennent pour l'essentiel d'expérimentation en laboratoire. Le transfert aux végétaux par dépôt foliaire est le mécanisme le plus pénalisant, les transferts par voie racinaire étant nettement plus faibles, d'un facteur 10 à 100 ; les transformations alimentaires réduisent enfin systématiquement l'activité présente dans les denrées commercialisées.

Par ailleurs, la rétention du cobalt par les sols est relativement importante, selon l'estimation fournie par les coefficients de partage. Cette affinité est en cohérence avec les facteurs de transferts racinaires relativement faibles mis en évidence par les expérimentations réalisées sur les végétaux.

\section{RÉFÉRENCES}

Alban L.A., Kubota J. (1960) A Study of extractable soil cobalt in soils of the Southeastern United States. Soil. Sci. Soc. Am. Proc. 24, L83-185.

Bachhuber H., Bunzl K., Schimmack W. (1986) Spatial variability of the distribution coefficients of ${ }^{137} \mathrm{Cs},{ }^{65} \mathrm{Zn},{ }^{85} \mathrm{Sr},{ }^{57} \mathrm{Co},{ }^{109} \mathrm{Cd},{ }^{141} \mathrm{Ce},{ }^{103} \mathrm{Ru},{ }^{95 \mathrm{mT}} \mathrm{Tc}$ and ${ }^{131} \mathrm{I}$ in a cultivated soil. Nucl. Techn. 72 (3), 359-371.

Baes C.F., Sharp R.D. (1981) Predicting radionuclide leaching from root zone soil for assessment applications. Trans. Am. Nucl. Soc. 38, 111-112.

Bunzl K., Schimmack W. (1988) Distribution coefficients of radionuclides in the soil; Analysis of the field variability. In: International conference on chemistry and migration behaviour of actinides and fission products in the geosphere (Migration '87). Munchen 14-18 Sept. 1987. Radiochim. Acta 44/45 (pt. 2) 355-360.

Colle C. (1994) The influence of processing on the radionuclide content of foodstuffs. In: Euratom Radiation Protection Programme 1990-1992, EUR 15295 1, pp. 636-639.

Colle C., Grauby A., Delmas J. (1991) Dépôt des radionucléides sur le sol et la végétation. in : Euratom Radiation Protection Programme 1985-1989, EUR 13268 1, pp. 887-892.

Colle C., Siclet F., Saas A. (1983) Principaux facteurs de transfert sol-plante obtenus en France pour des sols prélevés à proximité des sites PWR. In: Séminaire sur le transfert à l'homme des 
radionucléides libérés dans l'environnement par les installations nucléaires. Bruxelles, 17-21 Oct. 1983, pp. 371-387.

Coughtrey P.J., Thorne M.C. (1983) Radionuclide distribution and transport in terrestrial and aquatic ecosystems. Rotterdam : A.A. Balkema 2, pp. 157-179.

D'souza T.J., Mistry K.B. (1979) Uptake, distribution and metabolic fate of Fe59, Co58, Mn54 and Zn65 in plants and their mobility and availability to crops in typical black and laterite soils. In: Symposium de Colombo, 1978, Isotopes and radiation research on soil-plant relationships, pp. 407-425.

Kerpen W. (1986) Bioavailability of the radionuclides cesium-137, cobalt-60, manganese-54 and strontium-85 in various soils as a function of their soil properties. Methods applied and first results. In: Proceedings of an international seminar on application of distribution coefficients to radiological assessment models. Louvain-La-Neuve (Belgium). 7-11 Oct. 1985, pp. 322-335.

Lembrechts J.F., Van-Loon L.R., Van-Ginkel J.H., Desmet G.M. (1988) Interpretation of soil-to-plant transfer on the basis of soil solution chemical composition. In: Impact des accidents d'origine nucleaire sur l'environnement. Cadarache. Sept. 1988, 1 (D) pp. 169-178.

Lopez P.L., Graham E.P. (1972) Determination of labile pool of manganese, iron, zinc, cobalt and copper in deficient soils by isotopic exchange. Soil. Sci. 114, 295-299.

Loue A. (1986) Le cobalt. In: Les oligo-éléments en agriculture, Paris : Agri Nathan, pp. 194-196.

Pascal P. (1961) Nouveau traité de chimie minérale. Masson éditeur, Paris.

Real J. , Hugon J., Quinault J.M. (1983) Etude de la contamination foliaire des végétaux. Rapport CEA-SERE/EDF-SEI, Février 1983.

SchimmaCK W., Bunzl K., Dietl F., Klotz D. (1994) Infiltration of radionuclides with low mobility into a forest soil. Effect of the irrigation intensity. J. Env. Radioact. 24, 53-63.

Stefens W. et al. (1980) Lysimeter experiments on the transfer of Sr90, Cs137, Co60 and Mn54 from soil to plant. In: Actes du Ilème symposium de radioécologie de Cadarache, pp. 513-524.

Taylor R.M. (1968) The association of manganese and cobalt in soils : further observations. Soil. Sci. 19, 70-80.

Tiller K.G., Hodgson J.F., Peech S. (1962) Specific sorption of cobalt by soil clays. Soil. Sci 95, $392-$ 399.

Thibault D.H., Sheppard M.I., Smith P.A. (1990) A critical compilation and review of default soil/liquid partition coefficients $\mathrm{Kd}$ for use in radiological assessments. Atomic Energy of Canada Ltd., Pinawa, MB (Canada). Whiteshell Nuclear Research Establishment AECL10125.

Union Internationale des Radioécologistes (U.I.R.) (1989) IVth. Report of the working group soil to - plant transfer factors, Meeting in Guttannen, Switzerland, May 24-26. 